\title{
A study on the run length properties of the side sensitive group runs double sampling (SSGRDS) control chart
}

\author{
Nger Ling Chong ${ }^{1, *}$, Michael Boon Chong $\mathrm{Khoo}^{1}$, Zhi Lin Chong ${ }^{2}$ and Wei Lin Teoh ${ }^{3}$ \\ ${ }^{1}$ School of Mathematical Sciences, Universiti Sains Malaysia, 11800 Minden, Penang, Malaysia \\ ${ }^{2}$ Department of Physical and Mathematical Science, Faculty of Science, Universiti Tunku Abdul Rahman, 31900 Kampar, Perak, \\ Malaysia \\ ${ }^{3}$ School of Mathematical and Computer Sciences, Heriot-Watt University Malaysia, 62200 Putrajaya, Malaysia
}

\begin{abstract}
The side sensitive group runs double sampling (SSGRDS) chart incorporates the control charting concepts of the side sensitive group runs (SSGR) and double sampling (DS) charts. The SSGRDS chart which combines the efficiency of its basic charts is an effective approach to increase the speed of mean shift detection. The performance of the SSGRDS chart, based on the average number of observations to signal (ANOS), median number of observations to signal (MNOS) and percentiles of the number of observations to signal (PNOS) is investigated in this paper. Based on the results obtained, it is found that the SSGRDS chart becomes more sensitive in detecting mean shifts with an increase in the size of the process mean shift. With the use of MNOS and PNOS to measure the performance of the SSGRDS chart, the entire run length distribution is considered and this leads to a more complete understanding of the performance of the chart. The findings in this paper will provide a clearer picture on the run length properties of the SSGRDS chart which will facilitate practitioners in using the chart.
\end{abstract}

\section{Introduction}

Companies strive to manufacture products of high quality standards to meet the requirements of customers. The ability of a company to produce high quality products leads to a competitive advantage over other companies and is a primary factor in achieving customer satisfaction. Statistical Process Control (SPC) refers to the employment of statistical techniques to enhance the quality of products manufactured. Control chart is an SPC tool which is extensively adopted to monitor how a process changes with time in industries and nonmanufacturing sectors. The Shewhart $\bar{X}$ chart possesses the ability to identify large process mean shifts quickly. However, the main drawback of the Shewhart $\bar{X}$ chart is its lack of sensitivity towards small and moderate process mean shifts.

Among the strategies used to enhance the performance of the Shewhart $\bar{X}$ chart is the double sampling (DS) $\bar{X}$ control charting approach. The DS $\bar{X}$ chart was introduced by [1]. A modified version of the DS scheme that makes an out-of-control decision at the combined-sample stage with regard to information obtained from both samples was proposed by [2]. It was found that the DS procedure provides superior statistical efficiency without increasing sampling in comparison to the Shewhart $\bar{X}$ chart [2]. On the other hand, the DS procedure can also minimize sampling while maintaining the statistical efficiency. Meanwhile, [3] investigated the performance of the DS $\bar{X}$ chart under non-normality and found that the DS $\bar{X}$ chart surpasses the Shewhart $\bar{X}$ chart in detecting small mean shifts. Some of the literature on the DS $\bar{X}$ chart can be seen in [4-7].

[8] developed the group runs (GR) chart which is an integration of the Shewhart $\bar{X}$ chart and an extended version of the conforming run length (CRL) chart. In comparison to the synthetic and Shewhart $\bar{X}$ charts, the GR chart has a considerably lower out-of-control average time to signal $\left(\mathrm{ATS}_{1}\right)$. Additionally, [9] proposed a side sensitive group runs (SSGR) control chart by combining the GR chart with the side sensitivity approach. The SSGR chart outperforms the Shewhart $\bar{X}$, GR and synthetic charts. Other related studies on the SSGR chart can be found in [10-12].

Past research has been done with the purpose of developing alternative control charts that are superior in terms of efficiency in detecting shifts. In fact, a control chart with better sensitivity to shifts and has a faster detection speed will lead to more savings in cost. Hence, [13] introduced the side sensitive group runs double sampling (SSGRDS) chart which integrates the efficiency of both the DS and SSGR charts. Merging of control charting methods is an approach to produce an enhanced chart that surpasses the performances of its individual counterparts. In this paper, the SSGRDS chart is studied in terms of the average number of observations to signal (ANOS), median number of observations to signal (MNOS) and percentiles of the number of observations to signal (PNOS). The rest of this paper is as follows: Section 2 outlines the implementation of the SSGRDS

*Corresponding author: ngerlingc@gmail.com 
chart while Section 3 focuses on the performance measures. In Section 4, the results are shown. The run length properties are discussed in Section 5 and conclusions are deduced in Section 6.

\section{Implementation of the SSGRDS chart}

The SSGRDS chart is comprised of the DS sub-chart and an extended version of the CRL sub-chart. CRL is the number of sampling stages between two successive nonconforming sampling stages, which includes the last nonconforming sampling stage. The first and second samples are represented by $n_{1}$ and $n_{2}$, respectively. For the DS sub-chart, $L$ and $L_{2}$ denote the control limits of the first and second sampling stages while $L_{1}$ is the warning limit of the first stage. Let $I_{1}=\left[-L_{1}, L_{1}\right], I_{2}=\left[-L,-L_{1}\right) \cup$ $\left(L_{1}, L\right], I_{3}=(-\infty,-L) \cup(L,+\infty)$ and $I_{4}=\left[-L_{2}, L_{2}\right]$. Fig. 1 shows the schematic representation of the DS subchart. Meanwhile, $L_{3}$ is the lower limit of the CRL subchart. The implementation of the SSGRDS chart is as shown below [13]:

1. Compute the charting parameters $n_{1}, n_{2}, L, L_{1}, L_{2}$ and $L_{3}$.

2. A sample with $n_{1}$ observations is taken from a population and the sample mean $\bar{X}_{1, i}=\frac{\sum_{j=1}^{n_{1}} X_{1 j}}{n_{1}}$ is computed, where $X_{1 j}$, for $j=1,2, \ldots, n_{1}$, are the observations in the first sample of sampling stage $i$. This is followed by the computation of $Z_{1, i}=\frac{\bar{X}_{1, i}-\mu_{0}}{\sigma / \sqrt{n_{1}}}$.

3. Sampling stage $i$ is conforming if $Z_{1, i} \in I_{1}$. Then return to Step 2.

4. Sampling stage $i$ is non-conforming if $Z_{1, i} \in I_{3}$. Then, proceed to Step 9.

5. A second sample of size $n_{2}$ is taken from the same population as that of the first sample if $Z_{1, i} \in I_{2}$. Subsequently, $\bar{X}_{2, i}=\frac{\sum_{j=1}^{n_{2}} X_{2 j}}{n_{2}}$ is computed, where $X_{2 j}$, for $j=1,2, \ldots, n_{2}$, are the observations in the second sample of sampling stage $i$.

6. The overall sample mean, $\bar{X}_{i}=\frac{n_{1} \bar{X}_{1, i}+n_{2} \bar{X}_{2, i}}{n_{1}+n_{2}}$ and the $Z$ statistic, $Z_{i}=\frac{\bar{X}_{i}-\mu_{0}}{\sigma / \sqrt{n_{1}+n_{2}}}$ are computed for sampling stage $i$.

7. The sampling stage $i$ is conforming if $Z_{i} \in I_{4}$ and return to Step 2. Otherwise, the process is declared as non-conforming and proceed to Step 8 .

8. Calculate the $K_{m}$ value, which is the amount of sampling stages between the current (included in the count) and ending (excluded in the count) nonconforming sampling stages.

9. The process is out-of-control if $K_{1} \leq L_{3}$; or for $m>1$, $K_{m} \leq L_{3}$ and $K_{m+1} \leq L_{3}$ for the first time and the value of the $Z$ statistic corresponding to the $m$ th and $(m+1)$ th non-conforming sampling stages lies on the similar side of the target mean $\mu_{0}$.
10. An out-of-control point is signaled and corrective actions are taken to investigate the process and eliminate the assignable cause(s).

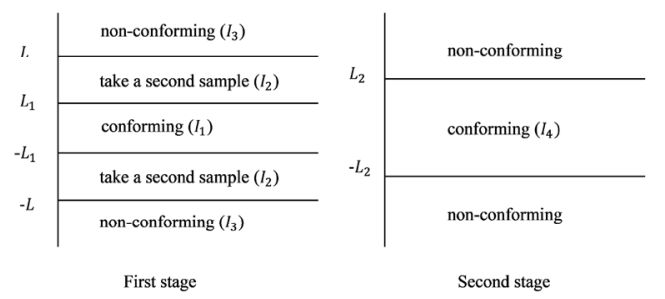

Fig. 1. Schematic representation of the DS sub-chart

\section{Performance Measures}

In this paper, ANOS is used to study the performance of the SSGRDS chart instead of the average run length (ARL) as the chart's sample size is not constant at each sampling stage (the sample size can be either $n_{1}$ or $n_{1}+$ $n_{2}$ ). In fact, ARL which represents the expected number of sampling stages required to signal an out-of-control condition is not as informative as ANOS that measures the actual number of observations needed by the chart to signal an out-of-control condition. MNOS refers to the median number of observations taken from the start of process monitoring until a signal is given by the chart. On the other hand, PNOS measures the percentiles of the number of observations required since the beginning of process monitoring until the chart signals an out-ofcontrol condition. Let the probability that the process is declared in-control be $P_{a}=P_{a 1}+P_{a 2}$ where $P_{a q}, q=1,2$ is the probability that the process is in-control at the $q$ th stage. It is given by [2] that

$$
\begin{aligned}
P_{a 1} & =\operatorname{Pr}\left(Z_{1} \in I_{1}\right) \\
& =\Phi\left(L_{1}+\delta \sqrt{n_{1}}\right)-\Phi\left(-L_{1}+\delta \sqrt{n_{1}}\right)
\end{aligned}
$$

where $\delta$ is the mean shift and $\Phi($.$) is the standard normal$ cumulative distribution function. Meanwhile [2],

$$
P_{a 2}=\operatorname{Pr}\left(Z \in I_{4} \text { and } Z_{1} \in I_{2}\right)=\int_{z \in I_{2}^{*}} P_{4} \phi(z) d z
$$

such that $\phi($.$) is the standard normal probability density$ function. Note that $I_{2}^{*}=\left[-L+\delta \sqrt{n_{1}},-L_{1}+\delta \sqrt{n_{1}}\right) \cup$ $\left(L_{1}+\delta \sqrt{n_{1}}, L+\delta \sqrt{n_{1}}\right.$ and

$$
P_{4}=\operatorname{Pr}\left(Z \in I_{4} \mid Z_{1}=z\right)
$$

Then ANOS can be computed as follows [13]:

$$
\mathrm{ANOS}=\frac{\left[1-\alpha(1-\alpha) A^{2}\right]\left(n_{1}+n_{2} P_{2}\right)}{A^{2}\left(1-P_{a}\right)[1+\alpha(1-\alpha)(A-2)]}
$$

where

$$
\begin{gathered}
P_{2}=\operatorname{Pr}\left(Z_{1} \in I_{2}\right) \\
A=\operatorname{Pr}\left(K_{m} \leq L_{3}\right)=1-(1-P)^{L_{3}}
\end{gathered}
$$

such that 


$$
P=1-\Phi\left(y_{s s g r}-\delta \sqrt{n}\right)+\Phi\left(-y_{s s g r}-\delta \sqrt{n}\right),
$$

and

$$
\begin{gathered}
\alpha=\operatorname{Pr}\left(Z_{1}>L \text { or }\left\{Z>L_{2} \text { and } Z_{1} \in\left(L_{1}, L\right)\right\} \mid\right. \text { non- } \\
\text { conforming sampling stage })
\end{gathered}
$$

such that $I_{2}^{\prime}=\left(L_{1}+\delta \sqrt{n_{1}}, L+\delta \sqrt{n_{1}}\right), r=\sqrt{n_{1}+n_{2}}$ and $c=\sqrt{\frac{n_{1}+n_{2}}{n_{2}}}$. Note that $y_{\text {ssgr }}$ is the width constant of the SSGR chart.

\section{Research Findings}

This section presents the findings of the performance measures ANOS, MNOS and PNOS of the SSGRDS chart. The in-control ANOS (ANOS $)_{0}$ is fixed as 370. In other words, it is assumed that when the process is incontrol, an average of 370 observations are needed before the SSGRDS chart signals a false alarm. It is worth noting that the $50^{\text {th }}$ percentile is equivalent to the MNOS. Simulation studies are conducted using the Statistical Analytical System (SAS) software to compute the out-ofcontrol ANOS, MNOS and PNOS denoted as $\mathrm{ANOS}_{1}$, $\mathrm{MNOS}_{1}$ and $\mathrm{PNOS}_{1}$, respectively. The various combinations of the optimal charting parameters $n_{1}, n_{2}, L, L_{1}, L_{2}, L_{3}$ are shown in Table 1 with the in-control average sample sizes, $\mathrm{ASS}_{0}=5$ and 10. The $\mathrm{ANOS}_{1}$ and MNOS $_{1}$ values are shown in Table 2 while the PNOS $_{1}$ values are enumerated in Table 3. Fig. 2 shows the comparison of the out-of-control ANOS and MNOS values when $\mathrm{ASS}_{0}=5$.

Table 1. Optimal charting parameters of the SSGRDS chart when $\operatorname{ANOS}_{0}=370$ for $\mathrm{ASS}_{0}=5$ and 10 (italicized)

\begin{tabular}{ccccccc}
\hline$\delta$ & $n_{1}$ & $n_{2}$ & $L$ & $L_{1}$ & $L_{2}$ & $L_{3}$ \\
\hline 0.2 & 3 & 10 & 3.2384 & 1.2781 & 1.5400 & 22 \\
& 5 & 15 & 3.0355 & 0.9626 & 1.3730 & 12 \\
\hline 0.6 & 3 & 4 & 3.2340 & 0.6726 & 1.4710 & 5 \\
& 7 & 4 & 3.0301 & 0.3154 & 1.2840 & 3 \\
\hline 1.0 & 4 & 2 & 3.2340 & 0.6726 & 1.3870 & 3 \\
& 7 & 4 & 3.0301 & 0.3154 & 1.1350 & 2 \\
\hline 1.4 & 4 & 2 & 3.2340 & 0.6726 & 1.2270 & 2 \\
& 6 & 5 & 3.0298 & 0.2502 & 1.1280 & 2 \\
\hline \multirow{2}{*}{1.8} & 3 & 3 & 3.2332 & 0.4290 & 1.2250 & 2 \\
& 5 & 6 & 3.0295 & 0.2073 & 1.1240 & 2 \\
\hline 2.0 & 3 & 3 & 3.2332 & 0.4290 & 1.2250 & 2 \\
& 4 & 7 & 3.0294 & 0.1769 & 1.1230 & 2 \\
\hline 2.6 & 3 & 3 & 3.2332 & 0.4290 & 1.2250 & 2 \\
& 3 & 8 & 3.0293 & 0.1542 & 1.2670 & 3 \\
\hline 3.0 & 2 & 4 & 3.2329 & 0.3170 & 1.3630 & 3 \\
& 2 & 9 & 3.0292 & 0.1366 & 1.2680 & 3 \\
\hline
\end{tabular}

Table 2. ANOS $_{1}$ and $\mathrm{MNOS}_{1}$ of the SSGRDS chart when $\mathrm{ASS}_{0}=5$ and 10

\begin{tabular}{|c|c|c|c|c|}
\hline \multirow{2}{*}{$\delta$} & \multicolumn{2}{|c|}{ ANOS $_{1}$} & \multicolumn{2}{c|}{ MNOS $_{1}$} \\
\cline { 2 - 5 } & $\mathrm{ASS}_{0}=5$ & $\mathrm{ASS}_{0}=10$ & $\mathrm{ASS}_{0}=5$ & $\mathrm{ASS}_{0}=10$ \\
\hline 0.2 & 85.70 & 75.33 & 45 & 45 \\
\hline 0.6 & 13.48 & 14.45 & 10 & 11 \\
\hline 1.0 & 6.79 & 9.76 & 6 & 11 \\
\hline 1.4 & 5.44 & 7.77 & 6 & 6 \\
\hline 1.8 & 4.64 & 5.98 & 6 & 5 \\
\hline 2.0 & 4.23 & 5.17 & 3 & 4 \\
\hline 2.6 & 3.30 & 3.57 & 3 & 3 \\
\hline 3.0 & 2.64 & 3.03 & 2 & 2 \\
\hline
\end{tabular}

Table 3. PNOS 1 of the SSGRDS chart when $\mathrm{ASS}_{0}=5$ and 10

\begin{tabular}{|c|c|c|c|c|c|c|c|c|}
\hline \multirow{3}{*}{$\delta$} & \multicolumn{7}{|c|}{ PNOS $_{1}$} \\
\cline { 2 - 9 } & \multicolumn{3}{|c|}{ ASS $_{0}=5$} & \multicolumn{4}{c|}{ ASS $_{0}=10$} \\
\cline { 2 - 9 } & $20^{\text {th }}$ & $40^{\text {th }}$ & $60^{\text {th }}$ & $80^{\text {th }}$ & $20^{\text {th }}$ & $40^{\text {th }}$ & $60^{\text {th }}$ & $80^{\text {th }}$ \\
\hline 0.2 & 19 & 35 & 56 & 94 & 25 & 35 & 55 & 90 \\
\hline 0.6 & 8 & 11 & 11 & 17 & 11 & 11 & 11 & 18 \\
\hline 1.0 & 6 & 6 & 6 & 6 & 7 & 11 & 11 & 11 \\
\hline 1.4 & 4 & 6 & 6 & 7 & 6 & 6 & 6 & 11 \\
\hline 1.8 & 3 & 3 & 6 & 6 & 5 & 5 & 5 & 5 \\
\hline 2.0 & 3 & 3 & 6 & 6 & 4 & 4 & 4 & 4 \\
\hline 2.6 & 3 & 3 & 3 & 3 & 3 & 3 & 3 & 3 \\
\hline 3.0 & 2 & 2 & 2 & 2 & 2 & 2 & 2 & 2 \\
\hline
\end{tabular}

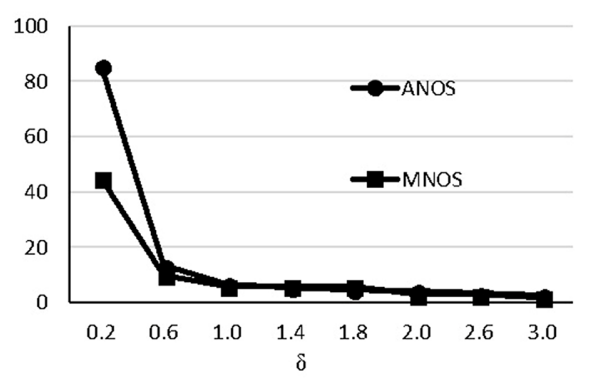

Fig. 2. Comparison of the out-of-control ANOS and MNOS values when $\mathrm{ASS}_{0}=5$.

\section{Run Length Properties}

In this paper, MNOS and PNOS are used besides ANOS to measure the performance of the SSGRDS chart. Based on Table 2 and Fig. 2, most of the $\mathrm{ANOS}_{1}$ values are greater than the $\mathrm{MNOS}_{1}$ values, indicating that the distribution of the number of observations is skewed to the right. However, the skewness level decreases as $\delta$ increases. Due to the skewness of the distribution of the number of observations, the interpretation of the SSGRDS chart's performance based on solely ANOS can be misleading. With MNOS and PNOS, the entire run length distribution is considered which provides more information on the performance of the SSGRDS chart. Additionally, median is less impacted by outliers compared to average, hence making it a more credible value to interpret a chart's performance. In Table 3 , the percentiles depict the confidence level of the occurrence 
of an out-of-control signal. As an illustration, when $\mathrm{ASS}_{0}$ $=10$ and $\delta=0.2$, we are $80 \%$ confident that an out-ofcontrol condition is signalled by the SSGRDS chart at the $90^{\text {th }}$ observation. It is also observed from Table 3 that the out-of-control condition is signaled earlier as $\delta$ increases and the out-of-control condition is signaled at the same observation for all percentiles when $\delta$ is large $(\delta \geq 2.6)$.

It can be seen from Tables 2 and 3 that $\mathrm{ANOS}_{1}$, $\mathrm{MNOS}_{1}$ and $\mathrm{PNOS}_{1}$ decrease as $\delta$ increases for $\mathrm{ASS}_{0}=5$ and 10. To illustrate, for $\mathrm{ASS}_{0}=5$, the $\mathrm{ANOS}_{1}\left(\mathrm{MNOS}_{1}\right)$ decrease from 85.70 (45) when $\delta=0.2$ to 2.64 (2) when $\delta$ $=3.0$. Similarly, for the $60^{\text {th }}$ percentile of $\mathrm{ASS}_{0}=5$, the $\mathrm{PNOS}_{1}$ decreases from 56 when $\delta=0.2$ to 2 when $\delta=3.0$. For an out-of-control process, it is desirable to have small ANOS, MNOS and PNOS values so that the process mean shift is detected quickly. Thus, the SSGRDS chart becomes more sensitive in detecting mean shifts as $\delta$ increases. Control charts are more likely to detect large process mean shifts swiftly compared to small process mean shifts as large shifts lead to a significant loss of quality.

\section{Conclusions}

This paper studies the performance of the SSGRDS chart in terms of the ANOS, MNOS and PNOS. Owing to the sample size of the SSGRDS chart that is not constant at each sampling stage, ANOS which is more informative as it evaluates the actual number of observations needed to signal an out-of-control condition is used instead of the typical performance measure ARL. Additionally, MNOS and PNOS are used as performance measures in this paper to assess the overall performance of the SSGRDS chart. As most of the $\mathrm{ANOS}_{1}$ values are greater than the $\mathrm{MNOS}_{1}$ values, the distribution of the number of observations is skewed to the right. Thus, the MNOS and PNOS are more suitable representations of the central tendency and are better indicators of the SSGRDS chart's performance. The results of this paper also show that the sensitivity of the SSGRDS chart increases with the shift size, which is justified by the need to detect large shifts that lead to a considerable loss of quality quickly.

This research is supported by the USM Research University Grant, Grant number: 1001.PMATHS.8011039 and funded by USM School of Mathematical Sciences (KPI MYRA PTJ), Grant number: 1001.PMATHS.AUPS001

\section{References}

1. R. Croasdale, Int. J. Prod. Res. 12, 585-592 (1974).

2. J.J. Daudin, J. Qual. Technol. 24, 78-87 (1992).

3. C.C. Torng and P.H. Lee. Commun. Stat. Simul. Comput. 38, 541-557 (2009).

4. L.F. Hsu, Int. J. Prod. Res. 42, 1043-1047 (2004).

5. M.B.C. Khoo, W.L. Teoh, P. Castagliola, and M.H. Lee, Int. J. Prod. Econ. 144, 345-357 (2013).

6. W.L. Teoh, M.B.C. Khoo., P. Castagliola, and S. Chakraborti, Comput. Ind. Eng. 67, 104-115 (2014).
7. M.B.C. Khoo, H.C. Lee, Z. Wu, C.H. Chen, and P. Castagliola, IIE Trans. 43, 23-38 (2010).

8. M.P. Gadre and R.N. Rattihalli, Econ. Qual. Contr. 19, 29-43 (2004).

9. M.P. Gadre and R.N. Rattihalli, Stat. Method Appl. 16, 27-37 (2007).

10. H.W. You, M.B.C. Khoo, P. Castagliola, and Y. Ou, Comput. Stat. 30, 1245-1278 (2015).

11. S.L. Lim, M.B.C. Khoo, W.C. Yeong, and M.H. Lee, Comput. Ind. Eng. 90, 314-325 (2015).

12. M.P. Gadre, K.A. Joshi, and R.N. Rattihalli, J. Appl. Stat. 37, 2073-2087 (2010).

13. M.B.C. Khoo, E.K. Tan, Z.L. Chong, and S. Haridy, Int. J. Prod. Res. 53, 4735-4753 (2015). 